Article

\title{
On the Complex and Hyperbolic Structures for the $(2+1)$-Dimensional Boussinesq Water Equation
}

\author{
Figen Özpinar ${ }^{1, *}$, Haci Mehmet Baskonus ${ }^{2}$ and Hasan Bulut ${ }^{3}$ \\ Received: 2 November 2015; Accepted: 10 December 2015; Published: 17 December 2015 \\ Academic Editor: Carlo Cattani \\ 1 Department of Office Management and Manager Assistant, Bolvadin Vocational School, \\ AfyonKocatepe University, Afyonkarahisar 03300, Turkey \\ 2 Department of Computer Engineering, Faculty of Engineering, Tunceli University, Tunceli 62100, Turkey; \\ hmbaskonus@gmail.com \\ 3 Department of Mathematics, Faculty of Science, Firat University, Elazig 23119, Turkey; hbulut@firat.edu.tr \\ * Correspondence: fozpinar@aku.edu.tr; Tel.: +90-272-612-6353
}

\begin{abstract}
In this study, we have applied the modified $\exp (-\Omega(\xi))$-expansion function method to the $(2+1)$-dimensional Boussinesq water equation. We have obtained some new analytical solutions such as exponential function, complex function and hyperbolic function solutions. It has been observed that all analytical solutions have been verified to the $(2+1)$-dimensional Boussinesq water equation by using Wolfram Mathematica 9. We have constructed the two- and three-dimensional surfaces for all analytical solutions obtained in this paper using the same computer program.
\end{abstract}

Keywords: the modified $\exp (-\Omega(\xi))$-expansion function method; the $(2+1)$-dimensional Boussinesq water equation; complex hyperbolic function solution; exponential function solution

\section{Introduction}

The $(2+1)$ Boussinesq equation was founded to describe some physical facts such as the propagation of small-amplitude long waves in shallow water in 1987 [1]. Some authors have investigated the physical and analytical structures of the $(2+1)$-dimensional Boussinesq water equation by using various methods [1-3]. Moleleki and Khalique have considered the simplest equation method for solving the $(2+1)$-dimensional Boussinesq equation [4]. Zhang, Meng, $\mathrm{Li}$, and Tian have studied the soliton resonance condition of the $(2+1)$-dimensional Boussinesq equation which is used to describe the propagation of gravity waves on the surface of water [5]. The homogeneous balance method has been successfully applied to the $(2+1)$-dimensional Boussinesq equation [6,7]. Allen and Rowlands have discussed the stability of solitary waves of the $(2+1)$-dimensional Boussinesq water equation and found that pulse-like solutions to the $(2+1)$-dimensional Boussinesq water equation are stable against linear perturbations [3]. The most general methods along this direction such as the $\exp (-\Phi(\eta))$-expansion method [8-10], the transformed rational function method [11], Bäcklund transformations [12], Frobenius integrable decompositions [13], and the multiple exp-functions method [14,15] have been applied to the various differential equations by Ma, Zhu, Huang and Zhang et al. Moreover, Wronskian solutions to the $(1+1)$-dimensional Boussinesq equation have been systematically presented in [16].

The main aim of this paper is to determine whether or not the new analytical method will be a powerful tool for obtaining new exponential, hyperbolic and complex analytical solutions to the $(2+1)$-dimensional Boussinesq water equation defined by [1-3]:

$$
u_{t t}-u_{x x}-u_{y y}-\left(u^{2}\right)_{x x}-u_{x x x x}=0
$$


Equation (1) is used to describe the propagation of gravity waves on the surface of water, the propagation of small-amplitude long waves in shallow water. More generally, Boussinesq equations arise relatively in fluid and solid mechanics [17-19].

\section{Fundamental Properties of Method}

The general properties of the modified $\exp (-\Omega(\xi))$-expansion function method (MEFM) are proposed in this section. MEFM is based on the $\exp (-\Omega(\xi))$-expansion function method [2,8-10]. In order to apply this method to the nonlinear partial differential equations, we consider it as follows:

$$
P\left(u, u_{x}, u_{y}, u_{t}, u_{x x}, u_{y y}, u_{t t}, \cdots\right)=0,
$$

where $u=u(x, y, t)$ is an unknown function, $P$ is a polynomial in $u(x, y, t)$ and its derivatives, in which the highest order derivatives and nonlinear terms are involved and the subscripts stand for the partial derivatives. The basic phases of the method are expressed as follows:

Step 1: Let us consider the following traveling transformation defined by

$$
u(x, y, t)=U(\xi), \quad \xi=k(x+y-c t)
$$

Using Equation (3), we can convert Equation (2) into a nonlinear ordinary differential equation (NODE) defined by:

$$
\operatorname{NODE}\left(U, U^{\prime}, U^{\prime \prime}, U^{\prime \prime \prime}, \cdots\right)=0,
$$

where NODE is a polynomial of $U$ and its derivatives and the superscripts indicate the ordinary derivatives with respect to $\xi$.

Step 2: Suppose the traveling wave solution of Equation (4) can be rewritten in the following manner:

$$
U(\xi)=\frac{\sum_{i=0}^{N} A_{i}\left[\exp (-\Omega(\xi)]^{i}\right.}{\sum_{j=0}^{M} B_{j}\left[\exp (-\Omega(\xi)]^{j}\right.}=\frac{A_{0}+A_{1} \exp (-\Omega)+\cdots+A_{N} \exp (\mathrm{N}(-\Omega))}{B_{0}+B_{1} \exp (-\Omega)+\cdots+B_{M} \exp (\mathrm{M}(-\Omega))^{-}}
$$

where $A_{i}, B_{j},(0 \leqslant i \leqslant N, 0 \leqslant j \leqslant M)$ are constants to be determined later, such that $A_{N} \neq 0, B_{M} \neq 0$, and $\Omega=\Omega(\xi)$ solves the following ordinary differential equation:

$$
\Omega^{\prime}(\xi)=\exp (-\Omega(\xi))+\mu \exp (\Omega(\xi))+\lambda .
$$

Equation (6) has the following solution families [8-10]:

Family 1: When $\mu \neq 0, \lambda^{2}-4 \mu>0$,

$$
\Omega(\xi)=\ln \left(\frac{-\sqrt{\lambda^{2}-4 \mu}}{2 \mu} \tanh \left(\frac{\sqrt{\lambda^{2}-4 \mu}}{2}(\xi+E)\right)-\frac{\lambda}{2 \mu}\right) .
$$

Family 2: When $\mu \neq 0, \lambda^{2}-4 \mu<0$,

$$
\Omega(\xi)=\ln \left(\frac{\sqrt{-\lambda^{2}+4 \mu}}{2 \mu} \tan \left(\frac{\sqrt{-\lambda^{2}+4 \mu}}{2}(\xi+E)\right)-\frac{\lambda}{2 \mu}\right) .
$$

Family 3: When $\mu=0, \lambda \neq 0$, and $\lambda^{2}-4 \mu>0$,

$$
\Omega(\xi)=-\ln \left(\frac{\lambda}{\exp (\lambda(\xi+E))-1}\right) .
$$


Family 4: When $\mu \neq 0, \lambda \neq 0$, and $\lambda^{2}-4 \mu=0$,

$$
\Omega(\xi)=\ln \left(-\frac{2 \lambda(\xi+E)+4}{\lambda^{2}(\xi+E)}\right)
$$

Family 5: When $\mu=0, \lambda=0$, and $\lambda^{2}-4 \mu=0$,

$$
\Omega(\xi)=\ln (\xi+E) \text {. }
$$

such that $A_{0}, A_{1}, A_{2}, \cdots A_{N}, B_{0}, B_{1}, B_{2}, \cdots B_{M}, E, \lambda, \mu$ are constants to be determined later. The positive integers $N$ and $M$ can be determined by considering the homogeneous balance between the highest order derivatives and the nonlinear terms occurring in Equation (5).

Step 3: Substituting Equations (6) and (7-11) into Equation (5), we get a polynomial of exp $(-\Omega(\xi))$. We equate all the coefficients of same power of $\exp (-\Omega(\xi))$ to zero. This procedure yields a system of equations which can be solved to find $A_{0}, A_{1}, A_{2}, \cdots A_{N}, B_{0}, B_{1}, B_{2}, \cdots B_{M}, E, \lambda$, $\mu$ with the aid of Wolfram Mathematica 9. Substituting the values of $A_{0}, A_{1}, A_{2}, \cdots A_{N}, B_{0}, B_{1}, B_{2}, \cdots B_{M}, E, \lambda, \mu$ in Equation (5), the general solutions of Equation (5) complete the determination of the solution of Equation (1).

\section{Applications}

In this sub-section of the study, we apply the above-mentioned method to the $(2+1)$-dimensional Boussinesq water equation [1-3] for obtaining new analytical solutions such as a new hyperbolic function solution and a complex function solution.

Example 1. When we consider the $(2+1)$-dimensional Boussinesq water equation along with Equations (3) and (5), we obtain the following nonlinear ordinary differential equation:

$$
\left(c^{2}-2\right) U-U^{2}-k^{2} U^{\prime \prime}=0,
$$

where $c, k$ are constants and $U=U(\xi)$. Using the balance principle for determining the relationship between $U^{\prime \prime}$ and $U^{2}$, we derive the following equation:

$$
N=M+2 .
$$

By using this relationship, we can attain some new analytical solutions for Equation (1) as follows:

Case 1: Let $M=1$ and $N=3$, and we can write;

$$
\begin{gathered}
U=\frac{A_{0}+A_{1} \exp (-\Omega)+A_{2} \exp (2(-\Omega))+A_{3} \exp (3(-\Omega))}{B_{0}+B_{1} \exp (-\Omega)}, \\
U^{\prime}=\frac{\left[A_{1} \exp (-\Omega)\left(-\Omega^{\prime}\right)+A_{2} \exp (2(-\Omega))\left(-2 \Omega^{\prime}\right)+A_{3} \exp (3(-\Omega))\left(-3 \Omega^{\prime}\right)\right]\left[B_{0}+B_{1} \exp (-\Omega)\right]}{\left[B_{0}+B_{1} \exp (-\Omega)\right]^{2}} \\
-\frac{\left[A_{0}+A_{1} \exp (-\Omega)+A_{2} \exp (2(-\Omega))+A_{3} \exp (3(-\Omega)]\left[B_{1} \exp (-\Omega)\left(-\Omega^{\prime}\right)\right]\right.}{\left[B_{0}+B_{1} \exp (-\Omega)\right]^{2}}=\frac{Y}{\Psi^{\prime}} \\
U^{\prime \prime}=\frac{Y^{\prime} \Psi-Y \Psi^{\prime}}{\Psi^{2}}
\end{gathered}
$$

where $A_{3} \neq 0$ and $B_{1} \neq 0$. Substituting Equations (14) and (15) in Equation (12), we get an equation including $\exp (-\Omega(\xi))$ and its various powers. Therefore, we have a system of equations from the coefficients of polynomial of $\exp (-\Omega(\xi))$. Solving this system of equations yields the following coefficients: 


\section{Case 1.1:}

$$
\begin{aligned}
& A_{0}=-6 k^{2} \mu B_{0}, A_{1}=\frac{A_{2} B_{0}+6 k^{2}\left(B_{0}^{2}-\mu B_{1}^{2}\right)}{B_{1}}, A_{2}=A_{2}, A_{3}=-6 k^{2} B_{1}, B_{0}=B_{0} \\
& \lambda=-\frac{A_{2}+6 k^{2} B_{0}}{6 k^{2} B_{1}}, c=\frac{\sqrt{\left(A_{2}+6 k^{2} B_{0}\right)^{2}+72 k^{2}\left(1-2 k^{2} \mu\right) B_{1}^{2}}}{6 k B_{1}}, B_{1}=B_{1}, k=k, \mu=\mu .
\end{aligned}
$$

\section{Case 1.2:}

$$
\begin{aligned}
& A_{0}=\frac{\left(\lambda^{2}+2 \mu\right) A_{3}}{6 B_{1}}, A_{1}=\frac{A_{3}}{6}\left(\lambda^{2}+2 \mu+\frac{6 \lambda B_{0}}{B_{1}}\right), A_{2}=A_{3}\left(\lambda+\frac{B_{0}}{B_{1}}\right), A_{3}=A_{3}, \\
& k=\frac{-i \sqrt{A_{3}}}{\sqrt{6 B_{1}}}, c=-\frac{\sqrt{\left(\lambda^{2}-4 \mu\right) A_{3}+12 B_{1}}}{\sqrt{6 B_{1}}}, B_{1}=B_{1}, \lambda=\lambda, B_{0}=B_{0}, \mu=\mu .
\end{aligned}
$$

\section{Case 1.3:}

$$
\begin{aligned}
& A_{0}=\frac{6 k^{2} B_{0}^{2}\left(B_{0}-\lambda B_{1}\right)}{B_{1}^{2}}, A_{1}=\frac{6 k^{2} B_{0}\left(B_{0}-2 \lambda B_{1}\right)}{B_{1}}, A_{2}=-6 k^{2}\left(B_{0}+\lambda B_{1}\right), A_{3}=-6 k^{2} B_{1}, \\
& \mu=\frac{B_{0}\left(-B_{0}+\lambda B_{1}\right)}{B_{1}^{2}}, B_{0}=B_{0}, B_{1}=B_{1}, c=-\frac{\sqrt{4 k^{2} B_{0}^{2}-4 k^{2} \lambda B_{0} B_{1}+\left(2+k^{2} \lambda^{2}\right) B_{1}^{2}}}{B_{1}}, \\
& k=k, \lambda=\lambda .
\end{aligned}
$$

\section{Case 1.4:}

$$
\begin{aligned}
& A_{0}=\frac{k^{2} B_{0}\left(2 B_{0}^{2}-2 \lambda B_{0} B_{1}-\lambda^{2} B_{1}^{2}\right)}{B_{1}^{2}}, A_{1}=k^{2}\left(-8 \lambda B_{0}+2 \frac{B_{0}^{2}}{B_{1}}-\lambda^{2} B_{1}\right), A_{2}=-6 k^{2}\left(B_{0}+\lambda B_{1}\right), \\
& A_{3}=-6 k^{2} B_{1}, \mu=\frac{B_{0}\left(-B_{0}+\lambda B_{1}\right)}{B_{1}^{2}}, c=-\frac{\sqrt{-4 k^{2} B_{0}^{2}+4 k^{2} \lambda B_{0} B_{1}+\left(2-k^{2} \lambda^{2}\right) B_{1}^{2}}}{B_{1}}, \\
& B_{0}=B_{0}, B_{1}=B_{1}, k=k, \lambda=\lambda .
\end{aligned}
$$

Four families of explicit and exact solutions contain solitary, periodic and new traveling wave solutions. Using coefficients of Equation (16) along with Equations (3) and (7) in Equation (14), we obtain a new hyperbolic function solution for Equation (1) as follows:

$$
u_{1}(x, y, t)=\frac{6 k^{2} \mu\left[\left(A_{2}+6 k^{2} B_{0}\right)^{2}-144 k^{4} \mu B_{1}^{2}\right] \sec h^{2}[f(x, y, t)]}{\left[A_{2}+6 k^{2} B_{0}-6 k^{2} B_{1} \sqrt{-4 \mu+\frac{\left(A_{2}+6 k^{2} B_{0}\right)^{2}}{36 k^{4} B_{1}^{2}}} \tanh [f(x, y, t)]\right]^{2}},
$$

where $f(x, y, t)=\frac{1}{2} \sqrt{-4 \mu+\frac{\left(A_{2}+6 k^{2} B_{0}\right)^{2}}{36 k^{4} B_{1}^{2}}}[E+k x+k y-m t],-4 \mu+\frac{\left(A_{2}+6 k^{2} B_{0}\right)^{2}}{36 k^{4} B_{1}^{2}}>0$, and $m=\frac{\sqrt{\left(A_{2}+6 k^{2} B_{0}\right)^{2}+72 k^{2}\left(1-2 k^{2} \mu\right) B_{1}^{2}}}{6 B_{1}}$. Substituting Equation (17) along with Equations (3) and (7) into Equation (14), we obtain the new complex hyperbolic function solution for the $(2+1)$-dimensional Boussinesq water equation as follows:

$$
u_{2}(x, y, t)=\frac{p A_{3}\left[\lambda^{2}-6 \mu+2 \lambda \sqrt{p} \tanh [f(x, y, t)]+\left(\lambda^{2}+2 \mu\right) \tanh ^{2}[f(x, y, t)]\right]}{6 B_{1}[\lambda+\sqrt{p} \tanh (f(x, y, t))]^{2}},
$$


where $p=\lambda^{2}-4 \mu, f(x, y, t)=\frac{\sqrt{p}}{12 B_{1}}\left(6 E-i \sqrt{A_{3}}\left(\sqrt{6} x+\sqrt{6} y+t \sqrt{p A_{3}+12 B_{1}}\right)\right)$, and $p>0$.

Consider using Equation (18) along with Equations (3) and (7) in Equation (14), we find another new hyperbolic function solution for Equation (1) as follows;

$$
u_{3}(x, y, t)=\frac{6 k^{2} \operatorname{sech}^{2}\left[\frac{\left(-2 B_{0}+\lambda B_{1}\right)}{2 B_{1}} f(x, y, t)\right] B_{0}\left(B_{0}-\lambda B_{1}\right)\left(-2 B_{0}+\lambda B_{1}\right)^{2}}{B_{1}^{2}\left[\lambda B_{1}+\left(-2 B_{0}+\lambda B_{1}\right) \tanh \left[\frac{\left(-2 B_{0}+\lambda B_{1}\right)}{2 B_{1}} f(x, y, t)\right]\right]^{2}},
$$

in which $f(x, y, t)=E+k\left(x+y+\frac{t}{B_{1}} \sqrt{4 k^{2} B_{0}^{2}-4 k^{2} \lambda B_{0} B_{1}+\left(2+k^{2} \lambda^{2}\right) B_{1}^{2}}\right), \frac{\left(-2 B_{0}+\lambda B_{1}\right)^{2}}{B_{1}^{2}}>0$.

Substituting Equation (19) along with Equations (3) and (7) into Equation (14), we find a new exponential function solution for Equation (1) as follows:

$$
u_{4}(x, y, t)=\frac{k^{2}\left(-2 B_{0}+\lambda B_{1}\right)^{2}}{B_{1}^{2}}\left(-1+\frac{6 B_{0}\left(B_{0}-\lambda B_{1}\right)}{-2 B_{0} \sinh [m f(x, y, t)]+\lambda B_{1} e^{m f(x, y, t)}}\right),
$$

in which $f(x, y, t)=(E+k(x+y)) B_{1}+k t \sqrt{-4 k^{2} B_{0}^{2}+4 k^{2} \lambda B_{0} B_{1}+\left(2-k^{2} \lambda^{2}\right) B_{1}^{2}}, m=\frac{\left(-2 B_{0}+\lambda B_{1}\right)}{2 B_{1}^{2}}$, $\frac{\left(-2 B_{0}+\lambda B_{1}\right)^{2}}{B_{1}^{2}}>0$.

Case 2: Letting $M=2$ and $N=4$, we can write the following:

$$
\begin{gathered}
U=\frac{A_{0}+A_{1} \exp (-\Omega)+A_{2} \exp (2(-\Omega))+A_{3} \exp (3(-\Omega))+A_{4} \exp (4(-\Omega))}{B_{0}+B_{1} \exp (-\Omega)+B_{2} \exp (2(-\Omega))}=\frac{\mathrm{Y}}{\Psi}, \\
U^{\prime}=\frac{\mathrm{Y}^{\prime} \Psi-\mathrm{Y} \Psi^{\prime}}{\Psi^{2}}=\frac{K}{T^{\prime}} \\
U^{\prime \prime}=\frac{\mathrm{K}^{\prime} \mathrm{T}-\mathrm{KT}^{\prime}}{\mathrm{T}^{2}},
\end{gathered}
$$

where $A_{4} \neq 0$ and $B_{2} \neq 0$. Substituting Equations (24) and (25) in Equation (12), we get an equation including $\exp (-\Omega(\xi))$ and its various powers. Therefore, we have a system of algebraic equations from the coefficients of the polynomial of $\exp (-\Omega(\xi))$. Solving this system of equations yields the following coefficients;

\section{Case 2.1:}

$$
\begin{aligned}
& A_{0}=\frac{\mu A_{4} B_{0}}{B_{2}}, A_{1}=\frac{A_{4}}{B_{2}}\left(\lambda B_{0}+\mu B_{1}\right), A_{2}=\frac{A_{4}}{B_{2}}\left(B_{0}+\lambda B_{1}+\mu B_{2}\right), A_{3}=A_{4}\left(\lambda+\frac{B_{1}}{B_{2}}\right), \\
& k=\frac{i \sqrt{A_{4}}}{\sqrt{6 B_{2}}}, c=-\frac{\sqrt{-\left(\lambda^{2}-4 \mu\right) A_{4}+12 B_{2}}}{\sqrt{6 B_{2}}}, A_{4}=A_{4}, B_{0}=B_{0}, B_{1}=B_{1}, \lambda=\lambda, \mu=\mu .
\end{aligned}
$$

\section{Case 2.2:}

$$
\begin{aligned}
& A_{0}=\frac{\left(\lambda^{2}+2 \mu\right) A_{3}}{6 B_{1}}, A_{1}=\frac{A_{3}}{6}\left(\lambda^{2}+2 \mu+\frac{6 \lambda B_{0}}{B_{1}}\right), A_{2}=A_{3}\left(\lambda+\frac{B_{0}}{B_{1}}\right), A_{3}=A_{3}, \\
& k=\frac{i \sqrt{A_{4}}}{\sqrt{6 B_{2}}}, c=-\frac{\sqrt{\left(\lambda^{2}-4 \mu\right) A_{4}+12 B_{2}}}{\sqrt{6 B_{2}}}, B_{0}=B_{0}, B_{1}=B_{1}, B_{2}=B_{2}, \mu=\mu, \lambda=\lambda .
\end{aligned}
$$


By using coefficients of Equation (26) along with Equations (3) and (7) in Equation (24), we find another complex hyperbolic function solution for Equation (1) as follows:

$$
u_{5}(x, y, t)=\frac{A_{4} \mu\left(-\lambda^{2}+4 \mu\right) \operatorname{sech}^{2}\left[\frac{1}{12 B_{2}} \sqrt{\left(\lambda^{2}-4 \mu\right)}\left(6 E B_{2}+i \sqrt{A_{4}} f(x, y, t)\right)\right]}{B_{2}\left[\lambda+\sqrt{\left(\lambda^{2}-4 \mu\right)} \tanh \left[\frac{1}{12 B_{2}} \sqrt{\left(\lambda^{2}-4 \mu\right)}\left(6 E B_{2}+i \sqrt{A_{4}} f(x, y, t)\right)\right]\right]^{2}},
$$

where $f(x, y, t)=\sqrt{6 B_{2}}(x+y)+t \sqrt{-\left(\lambda^{2}-4 \mu\right) A_{4}+12 B_{2}}$, and $\lambda^{2}-4 \mu>0$.

By considering using the coefficients of Equation (27) along with Equations (3) and (7) in Equation (24), we obtain another complex hyperbolic function solution for the $(2+1)$-dimensional Boussinesq water equation as follows:

$$
u_{6}(x, y, t)=\frac{p A_{4}\left(p-2 \mu+2 \lambda \sqrt{p} \tanh [K f(x, y, t)]+p \tanh ^{2}[K f(x, y, t)]\right)}{6 B_{2}[\lambda+\sqrt{p} \tanh [K f(x, y, t)]]^{2}},
$$

where $\left.f(x, y, t)=6 B_{2} E+i \sqrt{A_{4}}\left(\sqrt{6 B_{2}}(x+y)+t \sqrt{\left(\lambda^{2}-4 \mu\right) A_{4}+12 B_{2}}\right), K=\frac{1}{12 B_{2}} \sqrt{\left(\lambda^{2}-4 \mu\right.}\right)$ and $p=\lambda^{2}-4 \mu>0$.

\section{Physical Expressions and Discussions and Remarks}

In this subsection of the manuscript, we introduce some basic properties of the MEFM and the physical meaning of the complex, dark solitonand hyperbolic function solutions found for Equation (1) obtained in this paper.

MEFM is more comprehensive according to the $\exp (-\Omega(\xi))$-expansion method because MEFM includes one more parameter such as $M$. This gives many coefficients, which leads to many more traveling wave solutions as evidenced by the fact that we have obtained so many analytical solutions to the $(2+1)$-dimensional Boussinesq water equation for only $M=1$ and $N=3$. If we take $M=3$ and $N=5$, we can write the following equations:

$$
U=\frac{A_{0}+A_{1} \exp (-\Omega)+A_{2} \exp (-2 \Omega)+A_{3} \exp (-3 \Omega)+A_{4} \exp (-4 \Omega)+A_{5} \exp (-5 \Omega)}{B_{0}+B_{1} \exp (-\Omega)+B_{2} \exp (-2 \Omega)+B_{3} \exp (-3 \Omega)}=\frac{Y}{\Psi},
$$

and

$$
\begin{aligned}
& U^{\prime}=\frac{Y^{\prime} \Psi-\Psi^{\prime} Y}{\Psi^{2}}, \\
& U^{\prime \prime}=\frac{{Y^{\prime}}^{\prime} \Psi^{3}-\Psi^{2} Y^{\prime} \Psi^{\prime}-\left(\Psi^{\prime \prime} Y+\Psi^{\prime} Y^{\prime}\right) \Psi^{2}+2\left(\Psi^{\prime}\right)^{2} Y \Psi}{\Psi^{4}},
\end{aligned}
$$

where $A_{5} \neq 0, B_{3} \neq 0$. When we use Equations (30) and (32) in Equation (12), we obtain a system of algebraic equations. By solving this system via Wolfram Mathematica 9, we can obtain other analytical solutions which cannot be obtained by using only the $\exp (-\Omega(\xi))$-expansion method. Therefore, this procedure of Equation (6) will contribute to more analytical solutions and to a better understanding of engineering and physical problems along with new physical predictions.

To the best of our knowledge, when we conduct a comparison with analytical solutions obtained by Ma [11-15], we have obtained similar hyperbolic solutions under the terms of $M=1$ and $N=3$; moreover, we have found new complex hyperbolic function solutions by using MEFM.When we compare these analytical solutions with solutions obtained by Lai, Wu, Zhou [1], Alam, Hafez, Akbar, Roshid [2], and Allen, Rowlands [3], and Chen, Yan, Zhang [7], they are new and have not been submitted to literature previously. 
Secondly, hyperbolic functions are circular functions as well [20]. They arise in many problems of mathematics and mathematical physics. For instance, the hyperbolic sinearises in the gravitational potential of a cylinder. The hyperbolic cosine function is the shape of a hanging cable. The hyperbolic tangent arises in the calculation of and rapidity of special relativity. All three appear in the Schwarzschild metric using external isotropic Kruskal coordinates in general relativity [20]. The hyperbolic secant arises in the profile of a laminar jet. The hyperbolic cotangent arises in the Langevin function for magnetic polarization [20]. It is estimated that all these analytical solutions are related to such physical problems.

In consideration of the surfaces depicted here, shown in Figures 1-9 they have been constructed using suitable parameters. These values of parameters are consistent with the physical meaning of the problem.

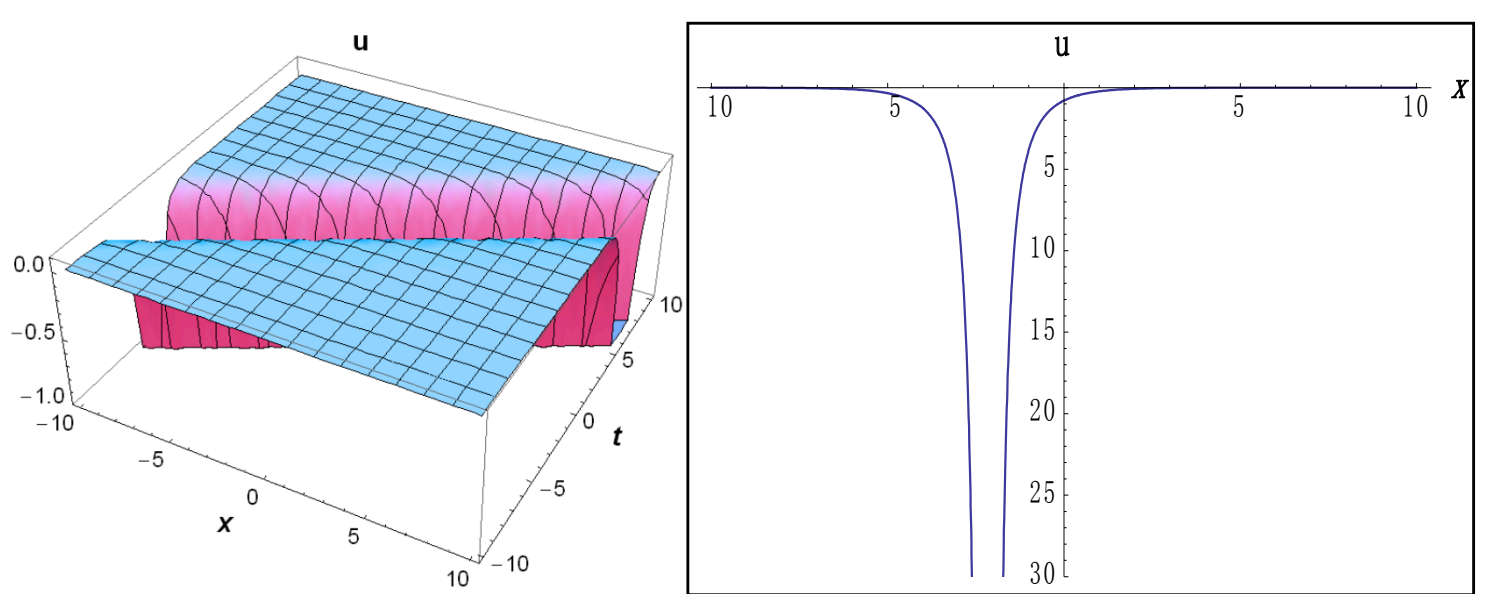

Figure 1. The 3D surfaces of the analytical solution, Equation (20), using the values $k=0.3, \mu=-3$, $A_{2}=1, B_{0}=-2, B_{1}=0.1, E=0.4, y=0.1,-10<x<10,-10<t<10$, and $t=0.001$ for $2 \mathrm{D}$ transect.

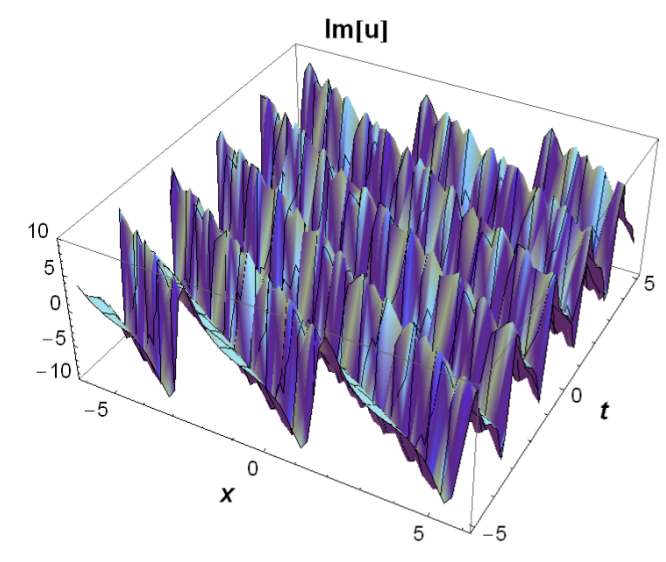

(a)

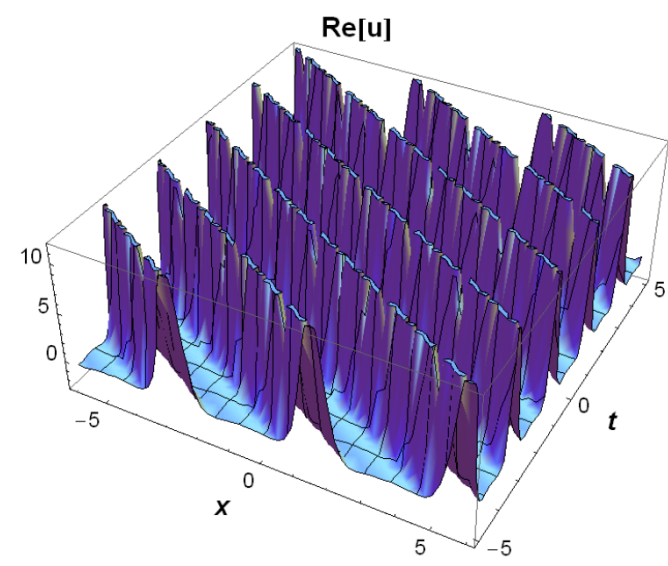

(b)

Figure 2. The 3D surfaces of the imaginary and real part of the analytical solution, Equation (21), using the values $\lambda=0.3, \mu=-0.3, A_{3}=1, B_{0}=-2, B_{1}=0.1, E=0.4, y=0.1,-6<x<6,-5<t<5$. (a) Imaginary part; (b) Real part. 


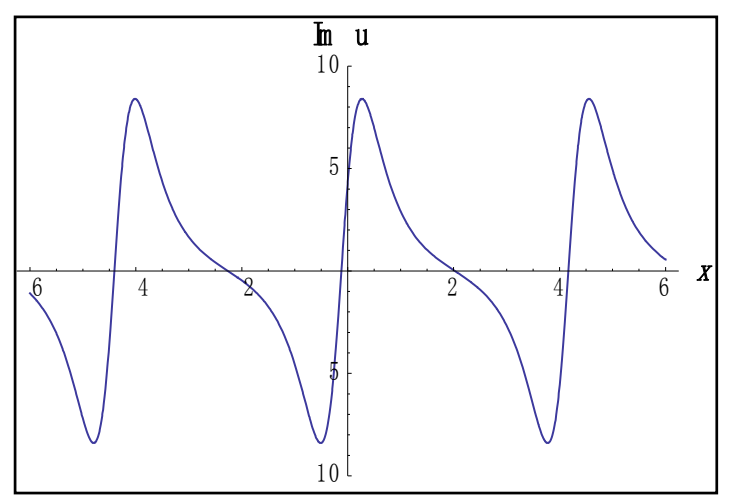

(a)

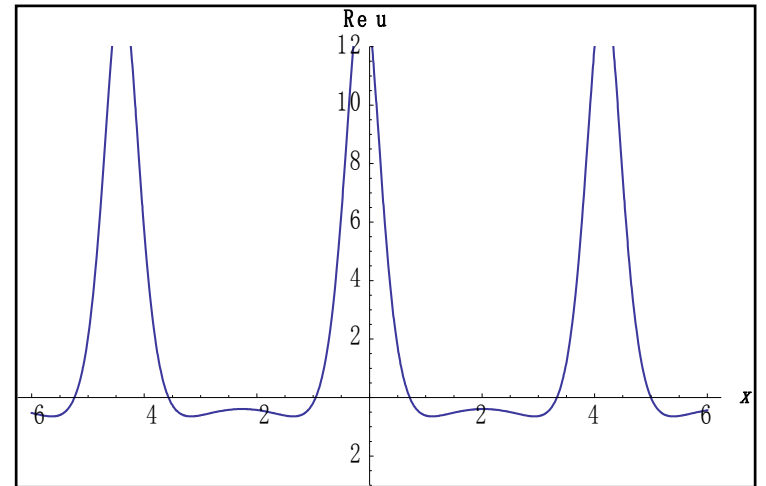

(b)

Figure 3. The $2 \mathrm{D}$ transect of the imaginary and real part of the analytical solution, Equation (21), using the values $\lambda=0.3, \mu=-0.3, A_{3}=1, B_{0}=-2, B_{1}=0.1, E=0.4, y=0.1, t=0.01,-6<x<6$. (a) Imaginary part; (b) Real part.

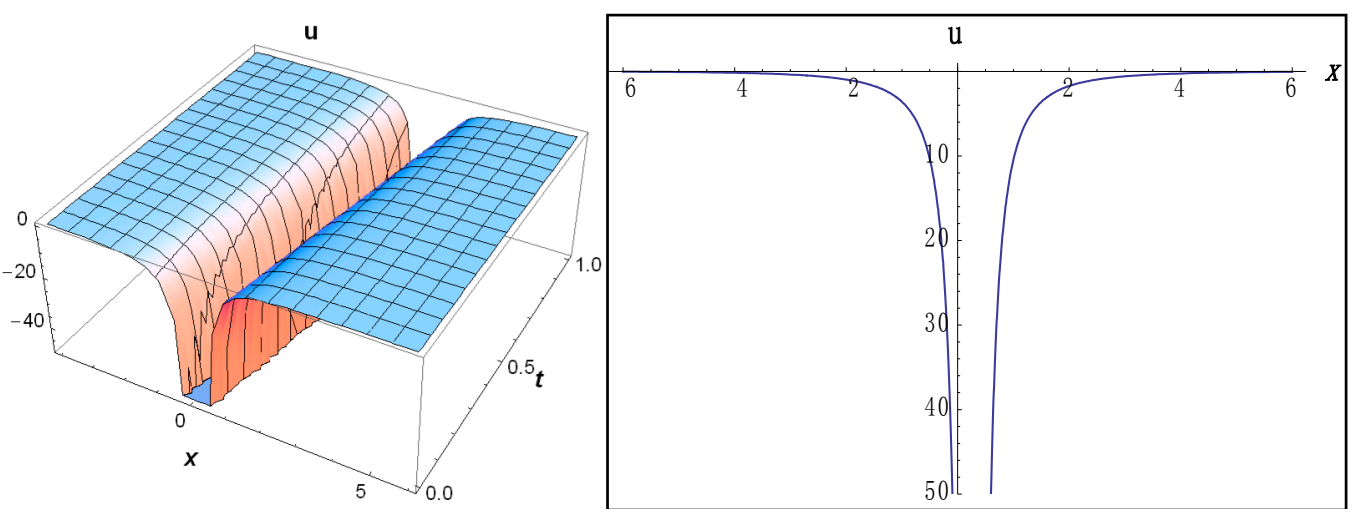

Figure 4. The $3 \mathrm{D}$ surface and $2 \mathrm{D}$ transect of the analytical solution, Equation (22), using the values $\lambda=0.3, E=-0.4, k=-0.5, B_{0}=-0.6, B_{1}=-0.7, y=-0.3,-4<x<6,0<t<1$, and $t=0.09$ for $2 \mathrm{D}$ transect.
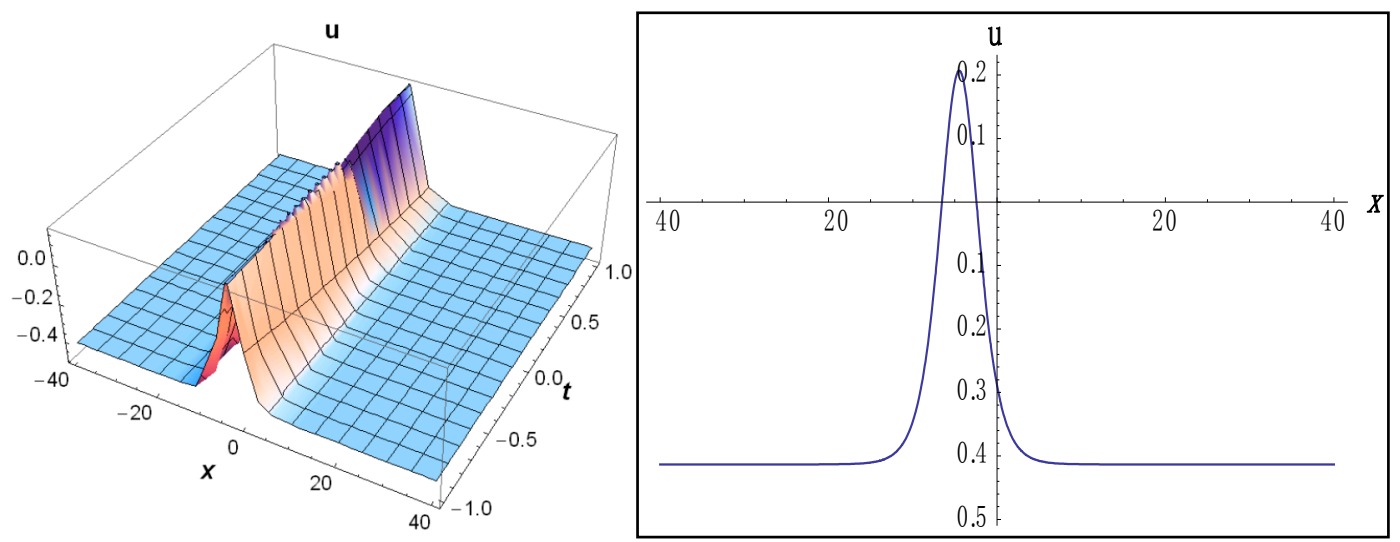

Figure 5. The $3 \mathrm{D}$ surface of the analytical solution, Equation (23), using the values $\lambda=3, E=0.4$, $k=0.5, B_{0}=0.6, B_{1}=0.7, y=2,-40<x<40,-1<t<1$, and $t=0.2$ for $2 \mathrm{D}$ transect. 


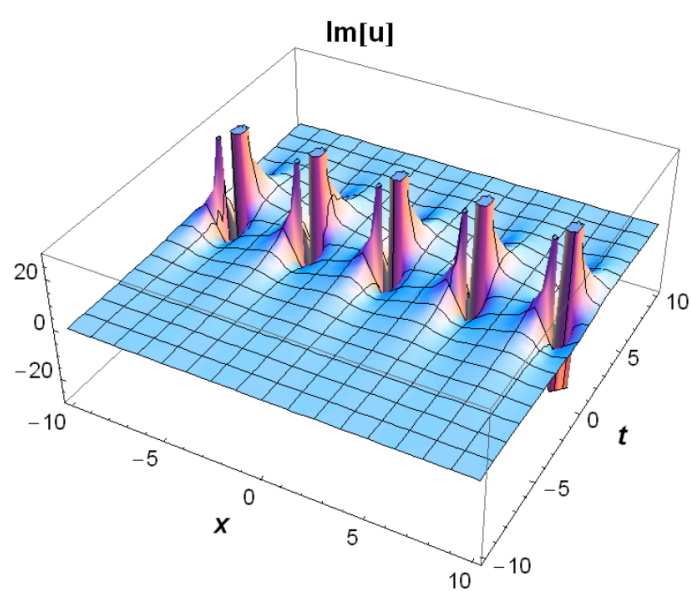

(a)

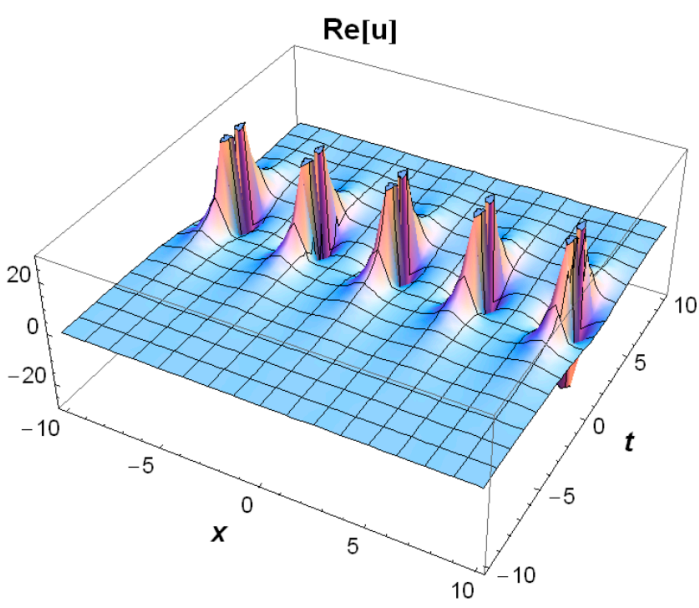

(b)

Figure 6. The $3 \mathrm{D}$ surfaces of the imaginary and real part of the analytical solution, Equation (28), using the values $\lambda=0.3, \mu=-0.3, A_{4}=1, B_{2}=0.1, E=0.4, y=0.1,-10<x<10,-10<t<10$. (a) Imaginary part; (b) Real part.

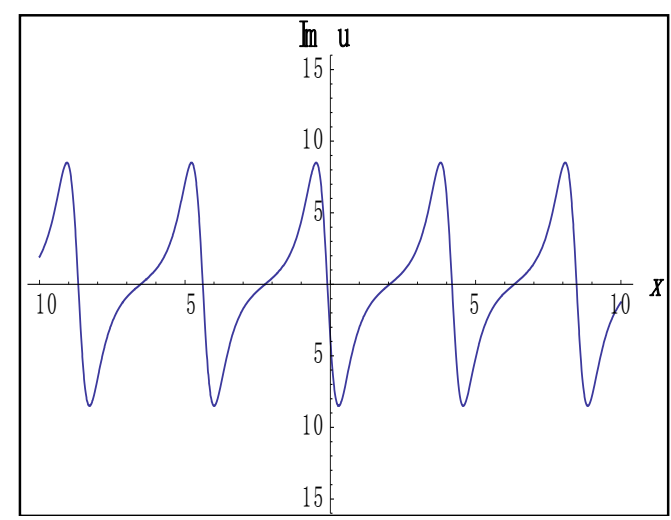

(a)

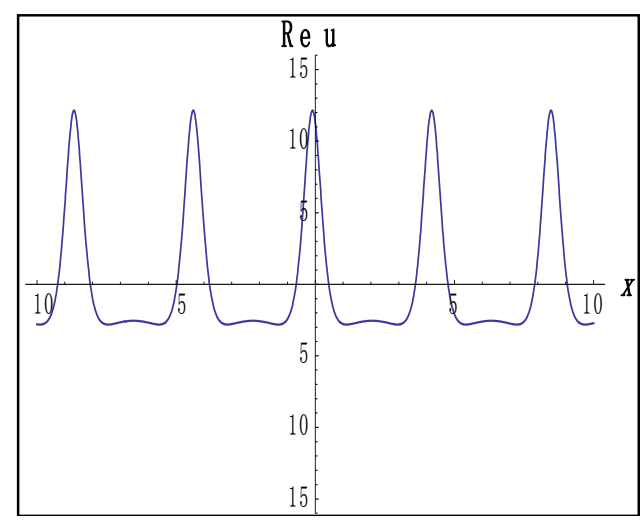

(b)

Figure 7. The 2D transect of the imaginary and real part of the analytical solution, Equation (28), using values $\lambda=0.3, \mu=-0.3, A_{4}=1, B_{2}=0.1, E=0.4, y=0.1, t=0.01,-10<x<10$. (a) Imaginary part; (b) Real part.

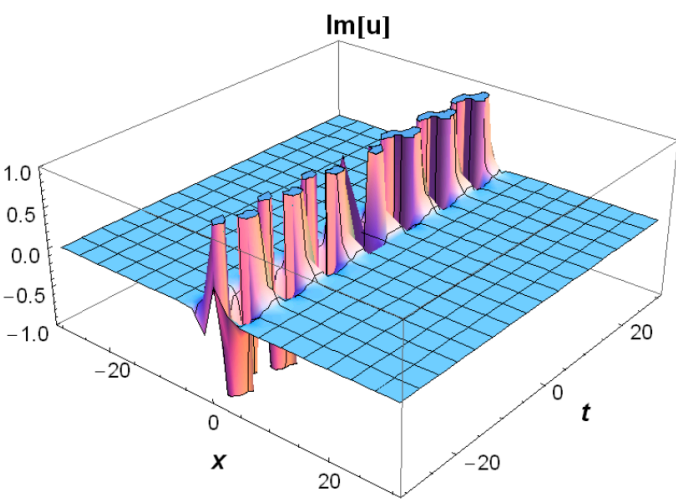

(a)

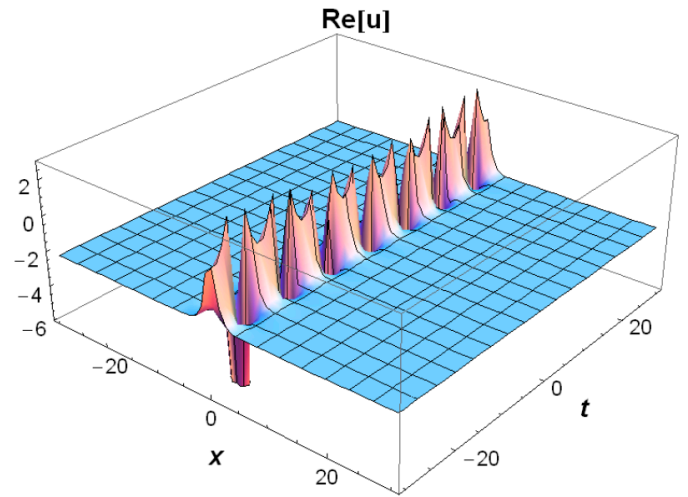

(b)

Figure 8. The 3D surfaces of the imaginary and real part of the analytical solution, Equation (29), using the values $\lambda=-0.3, \mu=-2, A_{2}=0.01, A_{4}=-1, B_{0}=0.3, B_{1}=0.5, B_{2}=0.6, E=-0.2, y=-0.1$, $-30<x<30,-30<t<30$. (a) Imaginary part; (b) Real part. 


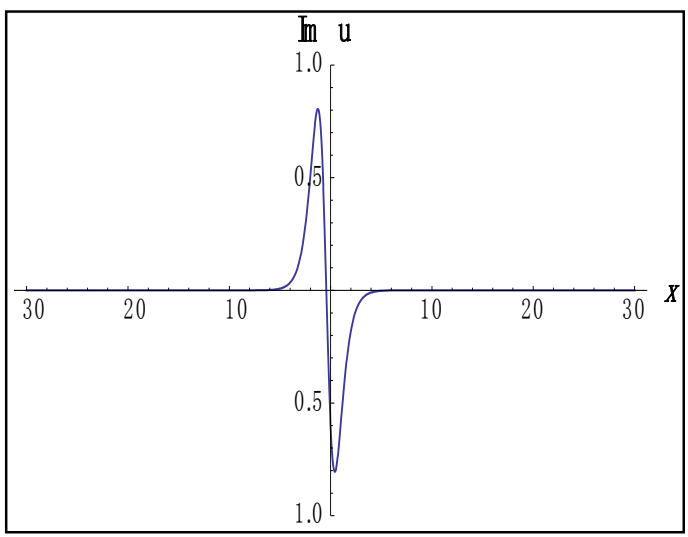

(a)

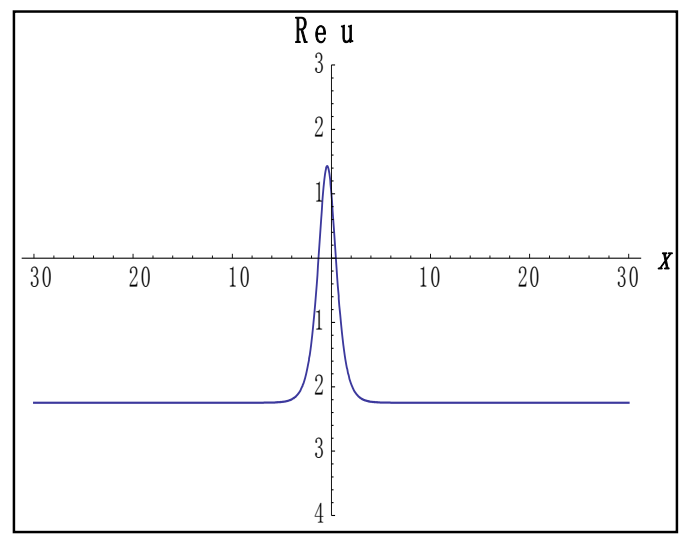

(b)

Figure 9. The $2 \mathrm{D}$ transects of the imaginary and real part of the analytical solution, Equation (29), using the values $\lambda=-0.3, \mu=-2, A_{2}=0.01, A_{4}=-1, B_{0}=0.3, B_{1}=0.5, B_{2}=0.6, E=-0.2$, $y=-0.1, t=5,-30<x<30$. (a) Imaginary part; (b) Real part.

\section{Conclusions}

In this paper we have applied the application of MEFM to the $(2+1)$-dimensional Boussinesq water equation. We have obtained some new analytical solutions such as exponential, complex and rational function solutions. We have observed that all analytical solutions obtained in this paper have verified to the Equation (1) by using Wolfram Mathematica 9. This method has provided many coefficients for Equations (14) and (24). Some of them have been considered in this paper to obtain new analytical solutions. If other coefficients are considered, of course, one can obtain different prototype solutions for Equation (1). Therefore, it can be said that this method is a powerful tool for obtaining solutions of the same type as Equation (1).

Acknowledgments: The authors would like to thank the reviewers for their valuable comments and suggestions to improve the present work.

Author Contributions: Figen Özpinar, Haci Mehmet Baskonus and Hasan Bulut have equally contributed to paper. All authors have read and approved the final version of the manuscript.

Conflicts of Interest: The authors declare no conflict of interest.

\section{References}

1. Lai, S.; Wu, Y.H.; Zhou, Y. Some physical structures for the $(2+1)$-dimensional Boussinesq water equation with positive and negative exponents. Comput. Math. Appl. 2008, 56, 339-345. [CrossRef]

2. Alam, M.N.; Hafez, M.G.; Akbar, M.A.; Roshid, H.O. Exact Solutions to the (2+1)-Dimensional Boussinesq Equation via $\exp (\Phi(\mathfrak{\eta}))$-Expansion Method. J. Sci. Res. 2015, 7. [CrossRef]

3. Allen, M.A.; Rowlands, G. On the transverse instabilities of solitary waves. Phys. Lett. A 1997, 235, 145-146. [CrossRef]

4. Moleleki, L.D.; Khalique, C.M. Solutions and Conservation Laws of a $(2+1)$-Dimensional Boussinesq Equation. Abstr. Appl. Anal. 2013, 2013. [CrossRef]

5. Zhang, H.; Meng, X.; Li, J.; Tian, B. Soliton resonance of the $(2+1)$-dimensional Boussinesq equation for gravity water waves. Nonlinear Anal. Real World Appl. 2008, 9, 920-926. [CrossRef]

6. Rady, A.S.A.; Osman, E.S.; Khalfallah, M. On soliton solutions of the $(2+1)$-dimensional Boussinesq equation. Appl. Math. Comput. 2012, 219, 3414-3419. [CrossRef]

7. Chen, Y.; Yan, Z.; Zhang, H. New explicit solitary wave solutions for $(2+1)$-dimensional Boussinesq equation and (3+1)-dimensional KP equation. Phys. Lett. A 2003, 307, 107-113. [CrossRef]

8. Roshid, H.O.; Rahman, M.A. The $\exp (-\Phi(\eta))$-expansion method with application in the $(1+1)$-dimensional classical Boussinesqequations. Results Phys. 2014, 4, 150-155. [CrossRef] 
9. Abdelrahman, A.E.; Zahran, E.H.M.; Khater, M.M.A. The $\exp (-\phi(\xi))$-Expansion Method and Its Application for Solving Nonlinear Evolution Equations Mahmoud. Int. J. Mod. Nonlinear Theory Appl. 2015, 4, 37-47. [CrossRef]

10. Hafez, M.G.; Alam, M.N.; Akbar, M.A. Application of the exp $(-\Phi(\eta))$-expansion Method to Find Exact Solutions for the Solitary Wave Equation in an Unmagnatized Dusty Plasma. World Appl. Sci. J. 2014, 32, 2150-2155.

11. Ma, W.-X.; Lee, J.-L. A transformed rational function method and exact solutions to the $(3+1)$ dimensional Jimbo-Miwa equation. Chaos Solitons Fractals 2009, 42, 1356-1363. [CrossRef]

12. Ma, W.X.; Fuchssteiner, B. Explicit and Exact Solutions to a Kolmogorov-Petrovskii-PiskunovEquation. Int. J. Nonlinear Mech. 1996, 31, 329-338. [CrossRef]

13. Ma, W.; Wu, H.; He, J. Partial differential equations possessing Frobeniusintegrable decompositions. Phys. Lett. A 2007, 364, 29-32. [CrossRef]

14. Ma, W.; Zhu, Z. Solving the $(3+1)$-dimensional generalized KP and BKP equations by the multiple exp-function algorithm. Appl. Math. Comput. 2012, 218, 11871-11879. [CrossRef]

15. Ma, W.-X.; Huang, T.; Zhang, Y. A multiple exp-function method for nonlinear differential equations and its application. Phys. Scri. 2010, 82, 065003. [CrossRef]

16. Ma, W.; Li, C.; He, J. A second Wronskian formulation of the Boussinesq equation. Nonlinear Anal. Theory Methods Appl. 2009, 70, 4245-4258. [CrossRef]

17. Daripa, P. Higher-order Boussinesq equations for two-way propagation of shallow water waves. Eur. J. Mech. B/Fluids 2006, 25, 1008-1021. [CrossRef]

18. Duruk, N.; Erkip, A.; Erbay, A.H. A higher-order Boussinesq equation in locally non-linear theory of one-dimensional non-local elasticity. IMA J. Appl. Math. 2009, 74, 97-106. [CrossRef]

19. Ali, A.; Kalisch, H. Mechanical balance laws for Boussinesq models of surface water waves. J. Nonlinear Sci. 2012, 22, 371-398. [CrossRef]

20. Weisstein, E.W. Concise Encyclopedia of Mathematics, 2nd ed.; CRC: New York, NY, USA, 2002.

(C) 2015 by the authors; licensee MDPI, Basel, Switzerland. This article is an open access article distributed under the terms and conditions of the Creative Commons by Attribution (CC-BY) license (http:/ / creativecommons.org/licenses/by/4.0/). 